\title{
Coexistence of plant species in a biodiversity hotspot is stabilized by competition but not by seed predation
}

\author{
Henning Nottebrock, Baptiste Schmid, Martina Treurnicht, Jörn Pagel, Karen J. Esler, \\ Katrin Böhning-Gaese, Matthias Schleuning and Frank M. Schurr
}

H. Nottebrock (henning@nottebrock.net), M. Treurnicht, J. Pagel and F. M. Schurr, Inst. of Landscape and Plant Ecology, Univ. of Hohenheim, August-von-Hartmann-Str. 3, DE-70599 Stuttgart, Germany. HN and FMS also at: Inst. des Sciences de l'Evolution, UMR 5554, Univ. Montpellier 2, Montpellier, France. - B. Schmid, K. Böhning-Gaese and M. Schleuning, Senckenberg Biodiversity and Climate Research Centre (BiK-F) and Senckenberg Gesellschaft für Naturforschung, Frankfurt am Main, Germany. KBG also at: Goethe University Frankfurt, Inst. for Ecology, Evolution and Diversity, Frankfurt (Main), Germany. - K. J. Esler, HN, BS and MT, Dept of Conservation Biology and Entomology and Centre for Invasion Biology, Stellenbosch University, Matieland, South Africa.

\begin{abstract}
Understanding the mechanisms of species coexistence is a key task for ecology. Recent theory predicts that both competition and predation (which causes apparent competition among prey) can either promote or limit species coexistence. Both mechanisms cause negative interactions between individuals, and each mechanism promotes stable coexistence if it causes negative interactions to be stronger between conspecifics than between heterospecifics. However, the relative importance of competition and predation for coexistence in natural communities is poorly known. Here, we study how competition and apparent competition via pre-dispersal seed predators affect the long-term fecundity of Protea shrubs in the fire-prone Fynbos biome (South Africa). These shrubs store all viable seeds produced since the last fire in fire-proof cones. Competitive effects on cone number and pre-dispersal seed predation reduce their fecundity and can thus limit recruitment after the next fire. In 27 communities comprising 49990 shrubs of 22 Protea species, we measured cone number and percone seed predation rate of 2154 and 1755 focal individuals, respectively. Neighbourhood analyses related these measures to individual-based community maps. We found that conspecific neighbours had stronger competitive effects on cone number than heterospecific neighbours. In contrast, apparent competition via seed predators was comparable between conspecifics and heterospecifics. This indicates that competition stabilizes coexistence of Protea species, whereas predispersal seed predation does not. Larger neighbours had stronger competitive effects and neighbours with large seed crops exerted stronger apparent competition. For $97 \%$ of the focal plants, competition reduced fecundity more than apparent competition. Our results show that even in communities of closely related and ecologically similar species, intraspecific competition can be stronger than interspecific competition. On the other hand, apparent competition through seed predators need not have such a stabilizing effect. These findings illustrate the potential of 'community demography', the demographic study of multiple interacting species, for understanding plant coexistence.
\end{abstract}

All species compete for resources with other species and they are themselves resources for predators (such as herbivores, carnivores, pathogens or parasites). Hence, it is not surprising that competition and predation are the most frequently investigated interactions in ecology (Chesson and Kuang 2008). Importantly, both competition and predation can play a key role for the coexistence of species on the same trophic level (Chase et al. 2002, Chesson and Kuang 2008).

Competition can either limit or promote stable coexistence of species on the same trophic level. The principle of competitive exclusion (Hardin 1960) states that if multiple species compete for one limiting resource in a closed, homogeneous environment, only the species that is able to maintain a stable population at the lowest resource level will survive in the long-term (Tilman 1982). However, competition can also promote the stable coexistence of multiple species. This is the case if intraspecific competition is stronger than interspecific competition, so that each species suppresses its own population growth more than that of other species (Chesson 2000). Stronger intraspecific competition results if conspecifics use more similar resources than heterospecifics or if their resource use overlaps more in space or time (Chesson 2000).

In plant communities, understanding species coexistence is particularly challenging. This is because most plant species use a small number of limiting resources (notably water, nutrients and light) in seemingly similar ways (Silvertown 2004). Various mechanisms have been proposed to explain how plant species can coexist in a local community by subdividing the commonly used resources (Silvertown 2004). For instance, the resource-ratio hypothesis states that plant species coexist if they have a tradeoff in their requirements 
for two essential resources and if the ratio at which these resources are supplied varies in space (Tilman 1982). Environmental variation at small spatial scales also plays a key role in the segregation of plant species along hydrological niche axes of water logging and drought stress (Silvertown et al. 1999, Araya et al. 2011). A prominent mechanism by which temporal variation in the environment can promote coexistence is the storage effect (Chesson 1994). The storage effect arises if species respond differentially to temporal variation, so that intraspecific competition is temporally more aggregated than interspecific competition and if species have means to store reproductive output during unfavourable times, for example as long-lived adult plants or in seed banks (Chesson 2000). These and other mechanisms of stable coexistence, such as the competition-colonization tradeoff (Tilman 1994), have in common that they cause intraspecific competition to be stronger than interspecific competition (Chesson 2000). In contrast, neutral models assume that competition is equally strong between conspecifics and heterospecifics. Under this assumption, stable coexistence is impossible and local diversity can only be maintained by immigration of species from the meta-community (Hubbell 2001).

The effects of predation on coexistence are in principle very similar to the effects of competition. This is because predation on prey individuals increases predator abundance which decreases the abundance of other prey. The resulting negative feedback has consequences for the population and community dynamics of prey that are largely analogous to those of competition for resources; hence, this predatormediated feedback is termed 'apparent competition' (Holt 1977). As in the case of competition, predation can either promote or limit the coexistence of prey species (Chesson and Kuang 2008). Predators that specialize on single prey species tend to promote stable coexistence because they cause apparent competition within species, but not between species. A prominent example is the Janzen-Connell hypothesis which states that the high diversity of tropical rainforest trees is due to spatial variation in the abundance of species-specific antagonists of seeds and seedlings (Janzen 1970, Connell 1971). However, even generalist predators shared by multiple prey species can stabilize coexistence as long as the resulting apparent competition is stronger within than between prey species (Chesson and Kuang 2008).

In plant communities, apparent competition within and between species can be mediated by herbivores, pathogens and seed predators (Hersh et al. 2012, Hulme 1996, 1998). Seed predation before or after seed dispersal is particularly important because it directly reduces plant reproductive output. Given that many plant populations are seed-limited (Turnbull et al. 2000), seed predation can have important effects on community dynamics. Hence, seed predators are likely to play an important role in restricting or enhancing coexistence in plant communities.

Community ecology has long regarded competition for resources as the primary interaction determining coexistence, with predation secondarily modifying the effects of resource competition (Chase et al. 2002). However, theoretical models suggest that competition and predation should be treated symmetrically. Coexistence can be either competition-based (if intraspecific competition is stronger than interspecifc competition), predation-based (if predator-mediated apparent competition is stronger within than between species) or jointly based on competition and predation (Chesson and Kuang 2008). Yet, there is a lack of studies quantifying the relative importance of competition and predation for intra- and interspecific interactions in natural communities.

Analyses of competition and apparent competition among plants have to account for the spatial and size structure of plant communities. Spatial community structure is important because the strength of interactions in sessile plant communities generally decreases with the distance between plants, so that plants mostly interact with their close neighbours (Stoll and Weiner 2000). Moreover, many plant communities comprise individuals of very different sizes, which are likely to have very different effects on their competitors and seed predators. Larger plants are stronger competitors because they take up more resources (Schenk 2006). Similarly, plants with larger seed crops offer more resources for predators and are thus expected to exert stronger apparent competition (Schnurr et al. 2002). Statistical neighbourhood models (Canham and Uriarte 2006) provide a flexible framework for quantifying how interactions among plants depend on plant traits (such as size and seed crop) and on the spatial distance between them. These models use spatially-explicit maps of communities and an explicit description of spatial interactions among plants to predict the performance of focal plants. Neighbourhood models have been used widely to study the effect of competition on the growth and mortality of long-lived plants (Uriarte et al. 2010, Kunstler et al. 2012). In communities of long-lived plants, it is particularly challenging to measure the relative importance of competition and predation. This is because competition and seed predation typically impact these communities on very different timescales: competition acts throughout the life cycle of a plant whereas seed predation is restricted to a relative short period of the life cycle. To compare the relative importance of competition and seed predation for coexistence, one therefore has to quantify their respective contributions to the lifetime fecundity of interacting plants. For most communities of long-lived plants this is a formidable task.

Here, we study how the long-term fecundity of long-lived plants is affected by competition and predator-mediated interactions within and between species. We studied these interactions in fire-prone shrubland communities in the South African Fynbos biome (a global biodiversity hotspot; Myers et al. 2000). These communities are dominated by serotinous shrubs of the genus Protea (Proteaceae) that form canopy seed banks, but no long-lived soil seed banks (Bond 1984, Rebelo 2001). Fire triggers seed release from fire-proof cones and subsequent seedling recruitment. Hence, the total fecundity of a plant since the last fire can be measured as the product of cone number, seed set per cone and seed predation rate per cone (Fig. 1a; Nottebrock et al. 2013). The majority of overstorey Protea species cannot survive fire as adults (Rebelo 2001); for members of these species, the total fecundity since the last fire is thus identical to their current lifetime fecundity (Nottebrock et al. 2013). Moreover, present-day community maps represent the longterm interaction neighbourhood of Protea plants because recruitment is limited to a short period after fire and the 
(a)

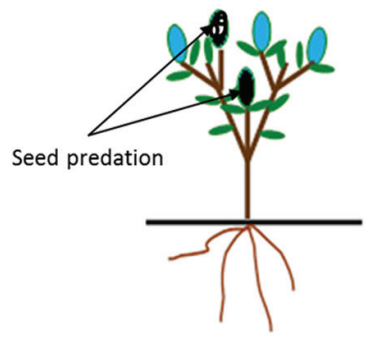

(b)

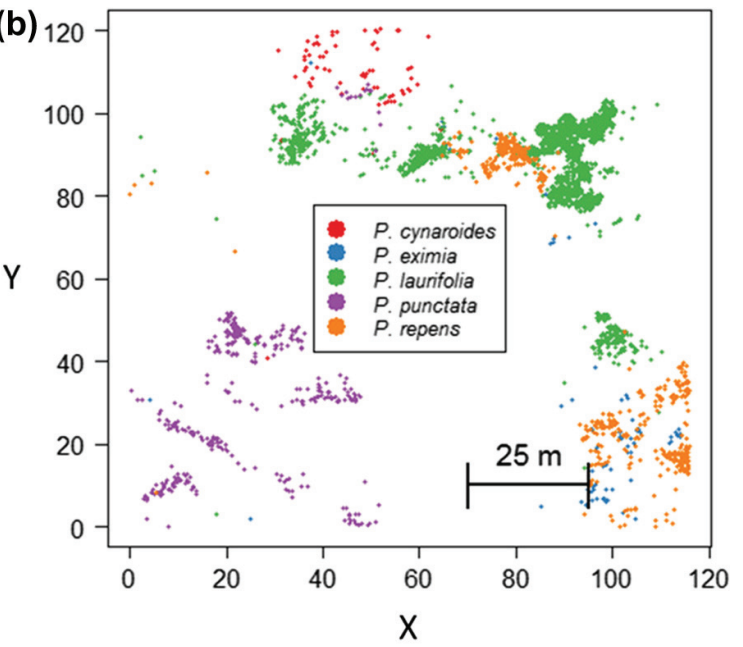

(c)

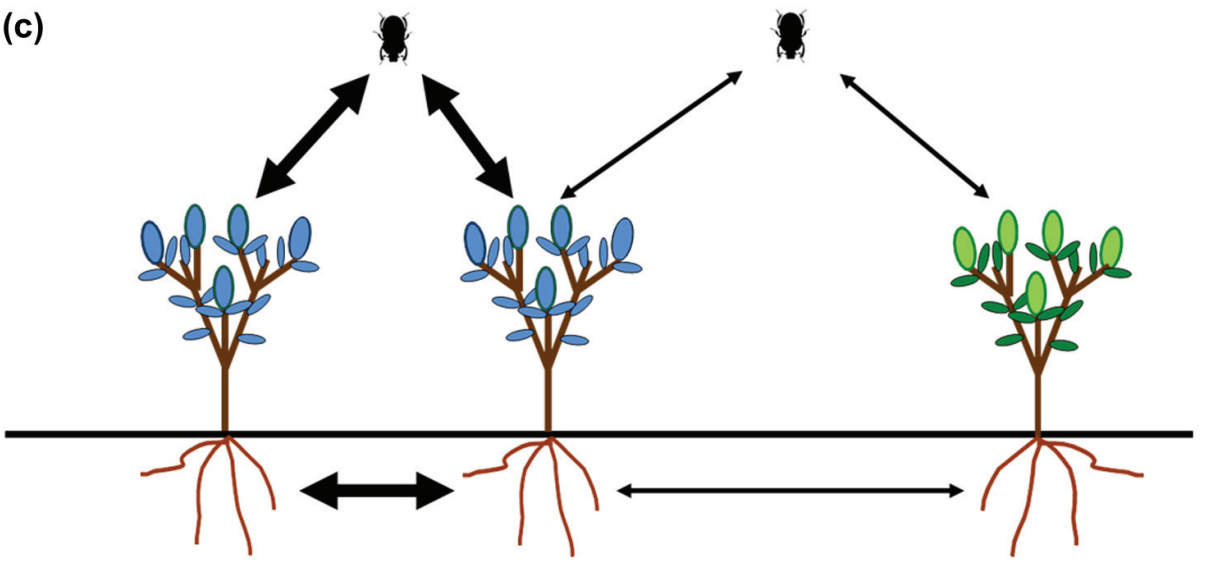

Figure 1. Effects of competition and apparent competition mediated by predators on the fecundity of serotinous Protea individuals. (a) The long-term fecundity of an individual depends on cone number and the rate of seed predation per cone. (b) Map of all Protea individuals on one of 27 study sites. (c) The fecundity of these individuals is affected by competition and apparent competition with conspecific and heterospecific neighbours; arrow sizes indicate hypothesized values of interaction strength between con- and heterospecific neighbours.

inter-fire mortality of successful recruits is generally very low (Bond et al. 1995). This facilitates analyses quantifying how conspecific and heterospecific neighbours affect the long-term fecundity of Protea individuals (Nottebrock et al. 2013). Making use of these features of serotinous shrub communities, we mapped 27 Protea communities at high spatial resolution and analysed the resulting maps of 49990 shrubs of 22 Protea species with neighbourhood models (Fig. 1). These analyses served to test four hypotheses: 1) competition is stronger between conspecifics than between heterospecifics; 2) apparent competition mediated by seed predators is stronger between conspecifics than between heterospecifics; 3 ) the size and seed crop of interacting plants determine the intensity of competition and apparent competition, respectively; and 4) competition and apparent competition have equally strong effects on the long-term fecundity of plants.

\section{Methods}

\section{Study system}

The genus Protea has high ecological and economic importance in the Fynbos biome (Schurr et al. 2012).
The overstorey Protea species studied here are all serotinous, broadleaf evergreen shrubs. They typically dominate on nutrient-poor soils (Groom and Lamont 2010) where they compete for soil nutrients and water rather than light (Cramer et al. 2014). Competition for soil resources is generally size-symmetric: larger plants take up more resources, but they do not have a disproportional advantage over smaller plants (other than in competition for light; Schenk 2006). A previous study by Nottebrock et al. (2013) suggests that resource competition among serotinous Fynbos Proteaceae primarily acts to decrease cone numbers per plant.

Pre-dispersal predation of the canopy seed banks of Protea is mostly caused by insects. Endophagous beetle and butterfly larvae, notably Sphenoptera spp. (Buprestidae), Genuchus hottentottus (Scarabaedidae), Argyroploce spp. (Olethreutidae), Tinea spp. (Tineidae) are major predators of Protea seeds (Coetzee and Giliomee, 1987a, b, Wright and Samways 1999).

\section{Study sites and mapping}

We selected 27 study sites of $120 \times 120 \mathrm{~m}$ that are dominated by Protea shrubs (Fig. 1b). Focal individuals for which we measured cone number and seed predation were located 
in the $100 \times 100 \mathrm{~m}$ core zones that were surrounded by $10 \mathrm{~m}$ wide buffer zones. We mapped all overstorey Protea plants (>30 cm height) on the study sites using differential GPS (median accuracy $20 \mathrm{~cm}$ ), and recorded their size (aboveground canopy height) and species identity. In very dense monospecific stands ( $>6$ individuals per $2 \mathrm{~m}^{2}$ ), we mapped the outline of the stand, recorded the plant density and then simulated plant locations within the stand according to a completely random spatial distribution with the observed densities. The sizes of these simulated plants were drawn from a stand-specific gamma distribution estimated by a maximum likelihood fit to the sizes of 30 plants measured per stand. In total, the resulting maps of our study communities describe the spatial location and size of 49990 individuals from 22 Protea species, of which $16.9 \%$ are simulated plants. The species composition and density of Protea plants varied widely both within and between the study sites (2-9 species per site, site-scale density: 0.003-0.767 plants $\mathrm{m}^{-2}$, density within $10 \mathrm{~m}$ around focal plants: 0-2.2 plants $\mathrm{m}^{-2}$ ). In 19 of 22 study species, adults cannot survive fire (so-called 'nonsprouters'; Rebelo 2001). For members of these species, the size of the canopy seed bank is therefore a direct measure of lifetime fecundity.

\section{Canopy seed banks of focal plants}

The size of canopy seed banks was measured on 2154 focal individuals within the core zone of the study sites. These focal individuals were selected non-randomly to increase variation in neighbourhood compositions. Accordingly, the proportion of heterospecific plants in $10 \mathrm{~m}$ radius around the focal plants varied from $0 \%$ to $100 \%$ with a mean of $59 \%$. For all focal plants, we counted the number of mature closed cones $(N)$ and for 1755 cone-bearing focal individuals, we harvested up to five mature cones per plant and cut the cones open with secateurs. Callipers were used to measure the cross-sectional diameter of each cone and the mean cross-sectional diameter of seeds from 33-365 cones per species. The total number of ovules per cone that could potentially set seed was then calculated by dividing the cross-sectional areas of cones by the cross-sectional area of a seed (determined as the mean per species and site population). To calculate seed crops, we also measured the mass of 284-1014 seeds per species using a high precision scale. The seed predation rate per harvested cone $(P)$ was measured as the mean proportion of the cross-sectional cone area that had been consumed by seed predators (Nottebrock et al. 2013).

\section{Neighbourhood analyses}

For neighbourhood analyses of the cone number and percone seed predation rate of focal plants, we used extensions of linear mixed models (package lme4, Bates et al. 2014) in R ver. 3.1.1 (<www.r-project.org >). We assumed Poisson errors for analyses of cone number and normal errors for analyses of logit-transformed seed predation rates (Warton and Hui 2011). The mixed models described interactions among plants by including neighbourhood indices as explanatory variables. For each focal plant $i$, these neighbourhood indices had the general form
$c_{\mathrm{i}}(\Omega, \mathrm{X})=\sum_{j \in \Omega} x_{j} \exp \left(-\frac{d_{i, j}^{2}}{\sigma^{2}}\right)$

where $\Omega$ defines the set of neighbours considered, e.g. all conspecific neighbours in a given radius around the focal plant. In the present analysis, we always considered neighbours in a radius of $10 \mathrm{~m} . x_{j}$ is a trait of neighbour plant $j$ (its size or potential seed crop). Alternatively, one can set $x_{j}=1$ for all neighbours, in which case the neighbourhood model describes simple density-dependence. Finally, the exponential term is a Gaussian interaction kernel that describes how neighbour effects decline with distance $d_{i, j}$ between the focal and the neighbour plant. The unknown spatial scaling parameter $\sigma$ is the distance at which the effect of a neighbour drops to $37 \%$ of the maximal value (this maximum is reached for $d_{i, j}=0$ ).

A model that includes such neighbourhood indices as explanatory variables is nonlinear in $\sigma$. Hence, it cannot directly be fitted with package lme4. We therefore used a two-level fitting procedure: one-dimensional optimization was used to find the value of $\sigma$ that maximizes the conditional $\log$-likelihood of the data given $\sigma$. For each value of $\sigma$, this conditional log-likelihood was obtained by calculating the neighbourhood variables and fitting the respective generalized linear mixed model.

To test our hypotheses, we fitted alternative neighbourhood models that contained different neighbourhood indices (Table 1). First, we tested whether conspecific neighbours had a stronger competitive effect on the cone number of focal plants than heterospecific neighbours. To this end, we fitted a density-dependent model (setting all $x_{j}=1$ in Eq. 1) which included two separate neighbourhood indices that were calculated for all conspecific and all heterospecific neighbours, respectively (model C2). To test whether effects of con- and heterospecific neighbours differ, we compared this model to a 'neutral' density-dependent model describing equal effects of con- and heterospecific neighbours ( $\mathrm{C} 1$, Table 1). This neutral model contained a single neighbourhood index that summed over all neighbours (irrespective of whether they were con- or heterospecific). Both models additionally included random effects of site and focal species identity on the intercept. Second, we fitted analogous density-dependent models for seed predation rate to test whether conspecific neighbours exert stronger apparent competition than heterospecific neighbours (P1 and P2; Table 1). Third, we tested whether accounting for traits of interacting plants (size or potential seed crop) improved models for cone number and seed predation, respectively. To this end, we fitted trait-dependent neighbourhood models that included an effect of the focal plant trait and that used the trait values of neighbours to calculate trait-dependent neighbourhood indices (Eq. 1). These trait-dependent neighbourhood models are thus weighted versions of the corresponding densitydependent models. They also contained random effects of site and focal species identity on the intercept and the focal trait slope. For each response variable, we fitted both a traitdependent model describing differential effects of con- and heterospecific neighbours (C4 and P4, respectively) and an alternative neutral model describing equal effects of all neighbours (C3 and P3, respectively). For trait-dependent 
Table 1. Comparison of alternative neighbourhood models for (a) cone number and (b) seed predation rate. Neighbour identity indicates whether models describe different effects of con- and heterospecifics or whether they describe identical effects of con- and heterospecifics. Neighbour effect indicates whether the strength of a neighbour's effect depends on plant size and potential seed crop, respectively, or whether the model describes density-dependence independent of plant or crop size. The best model (with lowest AIC) is highlighted in italics. We use conditional R²-values (Nakagawa and Schielzeth 2013).

\begin{tabular}{|c|c|c|c|c|c|}
\hline \multicolumn{6}{|c|}{ (a) Models for cone number } \\
\hline Model & Neighbour identity & Neighbour effect & DF & $\triangle \mathrm{AlC}$ & $\mathrm{R}^{2}$ \\
\hline C4 & con-versus heterospecific & size-dependent & 12 & 0 & 0.53 \\
\hline C3 & neutral & size-dependent & 11 & 6.7 & 0.53 \\
\hline $\mathrm{C} 2$ & con- versus heterospecific & density-dependent & 5 & 24953.6 & 0.52 \\
\hline $\mathrm{C} 1$ & neutral & density-dependent & 4 & 25356.6 & 0.52 \\
\hline $\mathrm{CO}$ & no neighbour effects & & 10 & 900.9 & 0.49 \\
\hline \multicolumn{6}{|c|}{ (b) Models for seed predation rate } \\
\hline Model & Neighbour identity & Neighbour effect & DF & $\triangle \mathrm{AIC}$ & $\mathrm{R}^{2}$ \\
\hline P4 & con- versus heterospecific & seed-crop-dependent & 12 & 2.0 & 0.16 \\
\hline P3 & neutral & seed-crop-dependent & 11 & 0 & 0.16 \\
\hline P2 & con- versus heterospecific & density-dependent & 6 & 3.3 & 0.15 \\
\hline P1 & neutral & density-dependent & 5 & 1.4 & 0.15 \\
\hline P0 & no neighbour effects & & 10 & 4.2 & 0.14 \\
\hline
\end{tabular}

analyses of seed predation, we calculated the potential seed crop of each plant as a measure of resource availability to seed predators. This potential seed crop was calculated as the product of cone number (predicted by the cone number model with the lowest AIC), potential seed set per cone and seed mass (see 'Canopy seed banks of focal plants' above). Fourth, we used the best model for each response variable (the model with the lowest AIC) to test whether competition and apparent competition differ in their effect on the long-term fecundity of Protea plants. The following section describes how these effects were quantified. Additionally, we assessed the importance of neighbourhood interactions by comparing the abovementioned trait- and density-dependent neighbourhood models to control models without neighbour effects ( $\mathrm{C} 0$ and $\mathrm{P} 0$, respectively). These control models were obtained by dropping the neighbourhood terms (Eq. 1) from the trait-based models.

\section{Quantifying effects of competition and predation on long-term plant fecundity}

The total fecundity of a plant since the last fire, $F$, is given by

$F=N \times S \times(1-P)$

where $N$ is the number of closed cones, $S$ is seed set per cone and $P$ is the seed predation rate. Since this expression is multiplicative, any change in cone number $(N)$ and in the proportion of seeds that are not consumed by predators $(1-P)$ causes a proportional change in fecundity. For each focal plant, we therefore used the best model for cone number to quantify the summed effect of competition on fecundity as $1-N_{n} / N_{0}$, where $N_{n}$ and $N_{0}$ are predicted cone numbers in the presence and absence of neighbours, respectively. Analogously, we calculated the summed effect of apparent competition mediated by predators as $1-\left(1-P_{n}\right) /$ $\left(1-P_{0}\right)$, where $P_{n}$ and $P_{0}$ are predation rates in the presence and absence of neighbours, as predicted by the best model for predation rate.

\section{Data deposition}

Data available from the Dataportal Senkenberg: doi: bikf.10023.1 <http://dataportal-senckenberg.de/database/ metacat/bikf.10023.1/bikf>. (Nottebrock et al. 2016b).

\section{Results}

The neighbourhood analyses of cone number and per-cone seed predation rate detected clear signs of both competition and apparent competition mediated by seed predators. All neighbourhood models for cone number $(\mathrm{C} 1-\mathrm{C} 4)$ estimated that neighbour effects decrease the cone number of focal plants, and all neighbourhood models for seed predation (P1-P4) estimated neighbours to increase the predation rate of focal plants (for examples see Fig. 2). Moreover, these neighbourhood models performed better than null models without neighbourhood effects ( $\mathrm{C} 0$ and $\mathrm{P} 0$, respectively).

As hypothesized, a density-dependent model for cone number estimated that conspecific neighbours have a much stronger competitive effect on cone number than heterospecific neighbours (model C2; Fig. 2a). This model, describing differential effects of con- and heterospecific neighbours, performed markedly better than an alternative model of neutral density-dependence in which con- and heterospecifics had the same competitive effect (model C1; likelihood ratio test: $\chi^{2}{ }_{1 \mathrm{DF}}=414.8, \mathrm{p}<0.001$; Table $1 \mathrm{a}$ ).

Contrary to our expectation, however, density-dependent models for seed predation showed that apparent competition was not stronger among conspecifics than among heterospecifics. A model in which the strength of apparent competition could differ between con- and heterospecifics (P2) estimated very similar effect sizes (Fig. 2a). Accordingly, this model did not perform better than the alternative model of neutral apparent competition $\left(\mathrm{P} 1 ; \chi^{2}{ }_{1 \mathrm{DF}}=0.03, \mathrm{p}>0.85\right.$; Table 1b).

Accounting for the size and potential seed crop of interacting plants improved neighbourhood models for both cone number and seed predation (Table 1). According to the 
(a)

Density-dependent models

Cone number

Predation

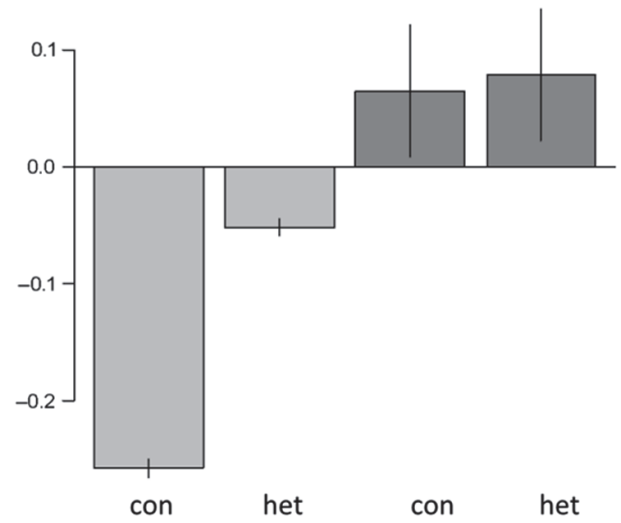

(b) Trait-dependent models

Cone number

Predation

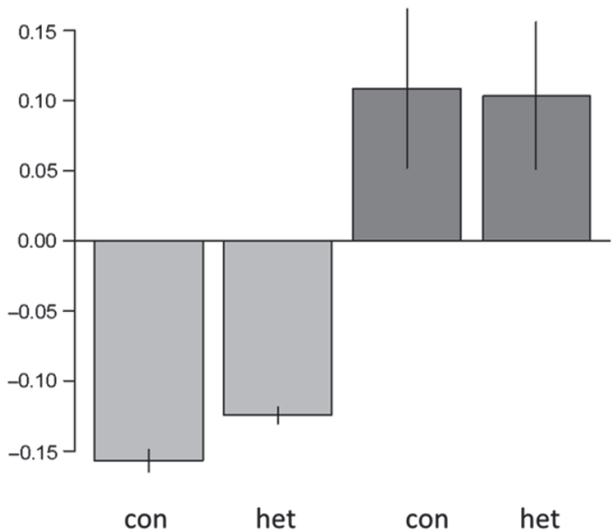

Figure 2. Effects of conspecific and heterospecific neighbours on cone number and seed predation of Protea individuals. The barplots show standardized neighbour effects and associated standard errors for (a) density-dependent and (b) trait-dependent neighbourhood models of cone number (light grey bars) and seed predation rate (dark grey bars). The trait-dependent models for cone number and seed predation accounted for the size and potential seed crop of interacting plants, respectively.

best model for cone number (C4), larger plants produced more cones, larger neighbours had a stronger competitive effect, and conspecific neighbours were stronger competitors than heterospecific neighbours (Fig. 2b). The best model for seed predation (P3) predicted that per-cone seed predation rates increased with the seed crop of focal plants and with the seed crop of their neighbours, but that neighbour effects did not differ between conspecifics and heterospecifics (Fig. $2 \mathrm{~b})$. These best, trait-dependent models for cone number and seed predation thus confirmed the findings of the simpler density-dependent models: conspecific neighbours had a stronger competitive effect on cone number than heterospecifics, but the effect of conspecifics on seed predation was comparable to that of heterospecifics. Overall, alternative models for seed predation showed smaller AIC differences than alternative models for cone number (Table 1). The best models of cone number and seed predation produced very similar estimates of the spatial scaling parameter $\sigma$, which suggests that competition and apparent competition act at similar spatial scales (Fig. 3).

We compared how competition and apparent competition affected the fecundity of those focal plants that were included in neighbourhood analyses of both cone number and seed predation rate. This comparison showed that plants suffering from strong competition also experienced strong apparent competition (one-sided Spearman's rank correlation test, $\rho=0.84, p<0.001$, Fig. 4). However, competition had markedly stronger effects than apparent competition (mean fecundity reductions: $10.7 \%$ and $2.6 \%$, respectively; one-sided paired Wilcoxon signed rank test, $\mathrm{V}=4566$, $\mathrm{p}<0.001$ ). In fact, competition effects were stronger than predation effects for $97 \%$ of the 1620 plants (Fig. 4).

\section{Discussion}

Our results show that neighbouring plants can reduce the long-term fecundity of Protea individuals both through direct competition and through apparent competition mediated by seed predators. As hypothesized, we found that conspecific neighbours have stronger competitive effects on cone number than heterospecific neighbours (Fig. 2). In contrast, we could not confirm the hypothesis that pre-dispersal seed predators cause apparent competition to be stronger between conspecifics than between heterospecifics (Fig. 2). This suggests that competition promotes the stable coexistence of Protea species, whereas pre-dispersal seed predation does not.

We also found support for the hypothesis that plant traits predict the intensity of interactions among plants. The competitive effects of neighbours increased with their size (as a proxy for resource acquisition; Schenk 2006) and predator-mediated effects of neighbours increased with their

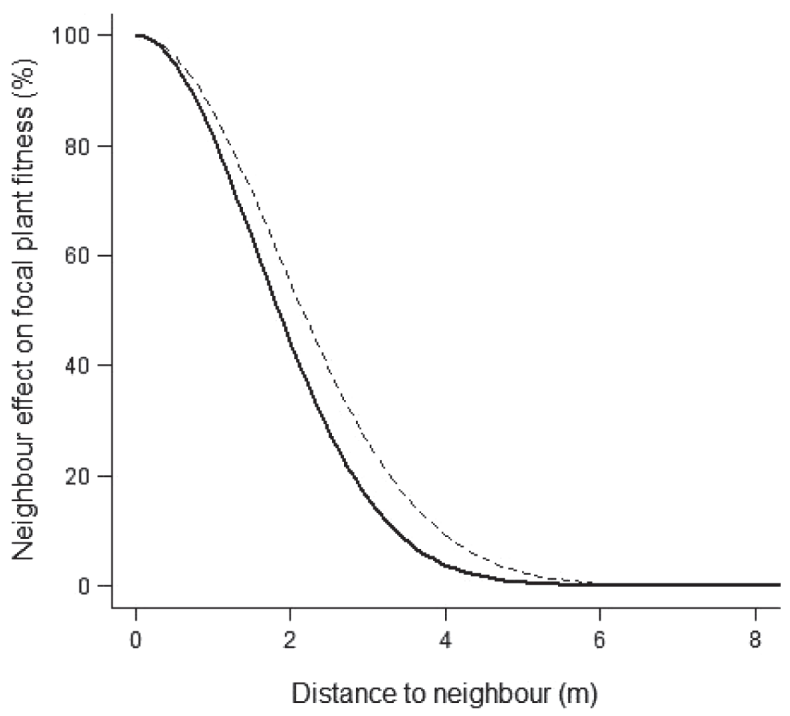

Figure 3. Distance dependence of neighbour effects estimated by the best neighbourhood models of cone number (solid line) and seed predation rate (dashed line). Neighbour effects are expressed relative to the maximal effect (as for a distance of 0 between a focal plant and its neighbour). 


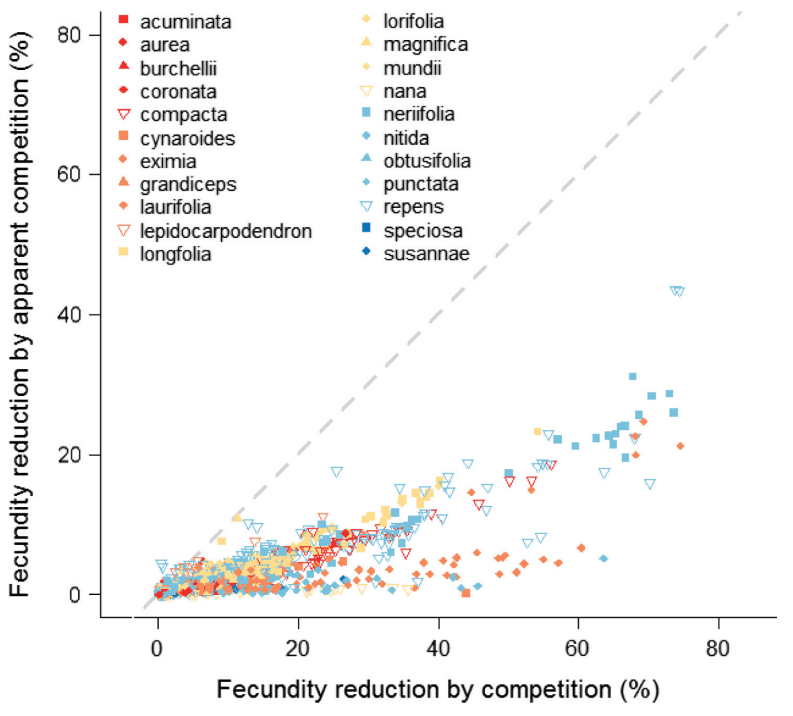

Figure 4. Relationship between the effects of competition and apparent competition on the fecundity of 1620 focal plants of 22 Protea species. These effects were calculated as the relative reduction of fecundity that is caused by all neighbouring plants (as predicted by the best neighbourhood models for cone number and seed predation, respectively; see Table 1). The dashed grey line is the $1: 1$ identity.

potential seed crop (as a measure of resource availability to seed predators). Simple traits measuring resource use by plants and resource availability to seed predators can thus explain the strength of biotic interactions in our study system. Finally, we rejected the hypothesis that competition and apparent competition via seed predators have similar effects on the fecundity of Protea. Instead, the effects of competition were much stronger than the effects of apparent competition (Fig. 4). It should be noted that the quantification of competitive effects was based on the best model for cone number which controlled for effects of focal plant size. The competitive effects on cone number estimated by this model do thus not incorporate competitive effects on focal plant size. Hence, the model may even underestimate the total amount of competition.

In the following, we first discuss mechanisms that may shape competition and predator-mediated interactions in Protea communities, and briefly highlight other processes that might affect the coexistence of Proted species. We end with a brief outlook on future studies in community demography.

\section{Mechanisms shaping competition and predator-mediated interactions in Protea communities}

Even though our study species are closely related and ecologically similar, we found that intraspecific competition is stronger than interspecific competition. This suggests that these species use resources at different spatial locations or at different times (Chesson 2000, Silvertown 2004). This result cannot be explained by simple spatial segregation of species (Pacala 1997), since our neighbourhood models detect stronger intraspecific competition while controlling for spatial community structure. However, fine-scale hydrological niche segregation may help to explain why conspecifics compete more intensively than heterospecifics. For another plant group in the Fynbos biome (Restionaceae), Araya et al. (2011) demonstrated fine-scale differentiation of hydrological niches that may reflect partitioning of space or water use over small scales via differences in rooting depth or phenology. In fact, phenological differentiation may play a key role for nutrient partitioning among Protea species. This is because the Mediterranean-type climate of Fynbos causes a temporal separation of optimal light and temperature conditions from peak water and nutrient availabilities, thereby creating opportunities for phenological differentiation in nutrient acquisition (Cramer et al. 2014). Interestingly, our study species show substantial phenological differentiation in flowering and cone production (Rebelo 2001, Nottebrock et al. 2016a). This might explain why we found that conspecifics have a stronger effect on cone numbers than heterospecifics. Alternatively, the temporal displacement of flowering phenologies is often interpreted as a consequence of interspecific competition for generalist pollinators (Devaux and Lande 2009). It will be interesting to explore to what extent phenological differentiation in flowering and cone production is driven by competition for nutrients, competition for pollinators and feedbacks between these two potential mechanisms.

While we found competition for resources to be stronger for conspecifics, the strength of apparent competition did not differ between conspecifics and heterospecifics. Although our large-scale analysis did not distinguish between different species of seed predators, this finding suggests that the main pre-dispersal seed predators of Protea are rather generalists that indiscriminately attack several host plant species. In fact, an extensive study of seed predator assemblages in Protea cones from six sites found nine predator species, which all attack a wide range of Protea species (Wright and Samways 1999).

The trait-based neighbourhood analyses suggest an interesting interaction between competition and seed predation: competition decreased individual seed crops in dense stands, which may reduce apparent competition. Nevertheless, we found a positive correlation between the strength of competition and apparent competition (Fig. 4). This is because on our study sites the effects of competition on seed crops were not overcompensating: the overall seed crop of dense stands was not smaller than that of sparse stands, even though individual seed crops were smaller. Interestingly, overcompensating density-dependence of seed crops has been documented in some Protea populations (Bond et al. 1995). It will be interesting to test whether the correlation between competition and apparent competition becomes negative under these circumstances.

\section{Alternative processes contributing to coexistence of Protea species}

The stabilizing effect of resource competition on coexistence of Protea species might be reinforced by other processes. A first option is interactions mediated by shared pollinators (Pauw 2013). Yet, an analysis of pollinator-mediated interactions in our study communities suggests that these interactions do not stabilize local coexistence (Nottebrock 
et al. 2016a). Second, coexistence may be promoted through post-dispersal seed predation (Hulme 1998). However, the seeds of our study species are simultaneously dispersed in vegetation-free post-fire landscapes (Schurr et al. 2005) where they face seed predation by generalist rodents (Bond 1984). While post-dispersal seed predation is thus unlikely to promote coexistence, it is conceivable that pathogens with a high host-specificity cause apparent competition to be stronger within than between species. Indeed, Proteaceae host a large number of fungal pathogens, some of which are assumed to be highly host-specific (Crous et al. 2011). In addition to these biotic interactions, a tradeoff between reproduction and survival may enhance coexistence. The genus Protea is a prime example for this tradeoff since it comprises both resprouters (with high fire survival and low fecundity) and nonsprouters (with low fire survival and high fecundity). However, models for Proteaceae communities suggest that local coexistence between resprouting and nonsprouting species only occurs under a restricted set of conditions (Groeneveld et al. 2002, Higgins et al. 2008). Finally, regional-scale processes might contribute to the maintenance of species richness in Protea meta-communities. Notably, resprouters and nonsprouters represent a competitioncolonization tradeoff (Schurr et al. 2007, Higgins et al. 2008), yet it remains to be tested whether this tradeoff is tight enough to enable the regional-scale coexistence of a large number of species (Tilman 1994). Regional-scale processes also figure prominently in the neutral theory (Hubbell 2001). However, analyses of rank-abundance data from Fynbos communities provide limited support for the neutral theory (Etienne et al. 2006). Our study adds to this by showing that competition in Fynbos communities is not neutral.

\section{Outlook}

This study highlights the potential and challenges of 'community demography', the demographic study of multiple interacting species. One of these challenges is the joint analysis of spatial and temporal variation in demography and biotic interactions. It seems particularly promising to combine the explicit description of demographic variation between individuals and years (Clark 2010) with the explicit description of direct and indirect spatial interactions between individuals (this study). Still, even without explicitly resolving variation between individuals and years, our neighbourhood analyses of long-term fecundity components lead to testable hypotheses. For instance, the hypothesized stabilizing effect of phenological differentiation in nutrient acquisition (Cramer et al. 2014) can be examined by combining field experiments and observations in Protea communities with tests for phenological overdispersion among neighbouring plants. Additionally, community demographic studies have the potential to identify determinants of biotic interactions that act across species and communities. For instance, we have identified the potential seed crop as an interaction currency (Kissling et al. 2012) that determines apparent competition between multiple plant species via shared seed predators. The identification of interaction currencies provides an alternative to the classical, yet idiosyncratic and unrealistic description of community dynamics in terms of interactions among species pairs (McGill et al. 2006, Clark 2010, Kissling et al. 2012). In megadiverse systems such as Fynbos, such generalizations across communities of different species compositions are urgently needed to understand and forecast community dynamics (Yates et al. 2010).

Acknowledgements - We thank a large number of field and lab assistants, especially Brummer Olivier. We were able to accomplish our study thanks to the support of private land-owners (Giel von Deventes, Flower Valley Conservation Trust; Mathia, Nayna and Walter Heidehof, Gansbaai; Bairie and Peter Gibson, Macially High Noon Farm G. and S. Moskovitz) and nature reserves (Grootbos, Fernkloof, Helderberg, Hottentots-Holland, Jonaskop, Limietberg, Mont-Rochelle).

Funding - This work was supported by the German Research Foundation [grant no. SCHU2259/3-1, SCHL 1934/1-1]. Field work was conducted under Cape Nature permit AAA005-002130028 .

\section{References}

Araya, Y. N. et al. 2011. - A fundamental, eco-hydrological basis for niche segregation in plant communities. - New Phytol. 189: 253-258.

Bates, D. et al. 2014. lme4: Linear mixed-effects models using Eigen and S4. - R package ver. 1.1-7, < http://CRAN.R-project. org $/$ package $=\operatorname{lme} 4>$. Last accessed 31 March 2015.

Bond, W. J. 1984. Fire survival of Cape Proteaceae-influence of fire season and seed predators. - Vegetatio 56: 65-74.

Bond, W. J. et al. 1995. Fire life-histories and the seeds of chaos. - Ecoscience 2: 252-260.

Canham, C. D. and Uriarte, M. 2006. Analysis of neighborhood dynamics of forest ecosystems using likelihood methods and modeling. - Ecol. Appl. 16: 62-73.

Chase, J. M. et al. 2002. The interaction between predation and competition: a review and synthesis. - Ecol. Lett. 5: 302-315.

Chesson, P. 1994. Multispecies competition in variable environments. - Theor. Popul. Biol. 45: 227-276.

Chesson, P. 2000. Mechanisms of maintenance of species diversity. - Annu. Rev. Ecol. Syst. 31: 343-366.

Chesson, P. and Kuang, J. J. 2008. The interaction between predation and competition. - Nature 456: 235-238.

Clark, J. S. 2010. Individuals and the variation needed for high species diversity in forest trees. - Science 327: 1129-1132.

Coetzee, J. H. and Giliomee, J. H. 1987a. Seed predation and survival in the infructescences of Protea repens (Proteaceae). - S. Afr. J. Bot. 53: 61-64.

Coetzee, J. H. and Giliomee, J. H. 1987b. Borers and other inhabitants of the inflorescences and infructescences of Protea repens in the Western Cape. - Phytophylactica 19: 1-6.

Connell, J. H. 1971. On the role of natural enemies in preventing competitive exclusion in some marine animals and in rain forest trees. - In: Den Boer, P. J. and Gradwell, G. R. (eds), Dynamics of populations. Center for Agric. Publ. and Documentation, Wageningen, pp. 298-310.

Cramer, M. et al. 2014. Plant ecophysiological diversity. - In: Allsopp, N. et al. (eds), Fynbos: ecology, evolution and conservation of a megadiverse region. Oxford Univ. Press, pp. 248-272.

Crous, P. W. et al. 2011. Fungal pathogens of Proteaceae. - Persoonia 27: 20-45.

Devaux, C. and Lande, R. 2009. Displacement of flowering phenologies among plant species by competition for generalist pollinators. - J. Evol. Biol. 22: 1460-1470. 
Etienne, R. S. et al. 2006. Comment on "Neutral ecological theory reveals isolation and rapid speciation in a biodiversity hot spot." - Science 311: 610-610.

Groeneveld, J. et al. 2002. A spatial model of coexistence among three Banksia species along a topographic gradient in fireprone shrublands. - J. Ecol. 90: 762-774.

Groom, P. K. and Lamont, B. B. 2010. Phosphorus accumulation in Proteaceae seeds: a synthesis. - Plant Soil 334: 61-72.

Hardin, G. 1960. The competitive exclusion principle. - Science 131: 1292-1298.

Hersh, M. H. et al. 2012. Evaluating the impacts of multiple generalist fungal pathogens on temperate tree seedling survival. - Ecology 93: 511-520.

Higgins, S. I. et al. 2008. Costs of persistence and the spread of competing seeders and sprouters. - J. Ecol. 96: 679-686.

Holt, R. D. 1977. Predation, apparent competition, and the structure of prey communities. - Theor. Popul. Biol. 12: $197-229$.

Hubbell, S. P. 2001. The unified neutral theory of biodiversity and biogeography. - Princeton Univ. Press.

Hulme, P. E. 1996. Herbivory, plant regeneration and species coexistence. - J. Ecol. 84: 609-615.

Hulme, P. E. 1998. Post-dispersal seed predation: consequences for plant demography and evolution. - Plant Ecol. Evol. Syst. 1: 32-46.

Janzen, D. H. 1970. Herbivores and the number of tree species in tropical forests. - Am. Nat. 104: 501-528.

Kissling, W. D. et al. 2012. Towards novel approaches to modelling biotic interactions in multispecies assemblages at large spatial extents. - J. Biogeogr. 39: 2163-2178.

Kunstler, G. et al. 2012. Competitive interactions between forest trees are driven by species' trait hierarchy, not phylogenetic or functional similarity: implications for forest community assembly. - Ecol. Lett. 15: 831-840.

McGill, B. J. et al. 2006. Rebuilding community ecology from functional traits. - Trends Ecol. Evol. 21: 178-185.

Myers, N. et al. 2000. Biodiversity hotspots for conservation priorities. - Nature 403: 853-858.

Nakagawa, S. and Schielzeth, H. 2013. A general and simple method for obtaining $R^{2}$ from generalized linear mixed-effects models. - Meth. Ecol. Evol. 4: 133-142.

Nottebrock, H. et al. 2013. Effects of intraspecific and community density on the lifetime fecundity of long-lived shrubs. - Plant Ecol. Evol. Syst. 15: 150-161.

Nottebrock, H. et al. 2016a. Sugar landscapes and pollinatormediated interactions in plant communities. - Ecography doi: $10.1111 /$ ecog.02441.

Nottebrock, H. et al. 2016b. Data from: Coexistence of plant species in a biodiversity hotspot is stabilized by competition but not by seed predation. - < http://dataportal-senckenberg.de/ database/metacat/bikf.10023.1/bikf $>$.

Pacala, S. W. 1997. Dynamics of plant communities. - In: Crawley, M. C. (ed.), Plant ecology. Blackwell, pp. 532-555.

Pauw, A. 2013. Can pollination niches facilitate plant coexistence? - Trends Ecol. Evol. 28: 30-37.

Rebelo, T. 2001. Proteas: a field guide to the Proteas of South Africa. - Fernwood Press, Vlaeberg.

Schnurr, J. L. et al. 2002. Direct and indirect effects of masting on rodent populations and tree seed survival. - Oikos 96: 402-410.

Schurr, F. M. et al. 2005. A mechanistic model for secondary seed dispersal by wind and its experimental validation. - J. Ecol. 93: 1017-1028.

Schurr, F. M. et al. 2007. Colonization and persistence ability explain the extent to which plant species fill their potential range. - Global Ecol. Biogeogr. 16: 449-459.

Schurr, F. M. et al. 2012. Fynbos Proteaceae as model organisms for biodiversity research and conservation. - S. Afr. J. Sci. 108: $12-16$.

Schenk, H. J. 2006. Root competition: beyond resource depletion. - J. Ecol. 94: 725-739.

Silvertown, J. 2004. Plant coexistence and the niche. - Trends Ecol. Evol. 19: 605-611.

Silvertown, J. et al. 1999. Hydrologically defined niches reveal a basis for species-richness in plant communities. - Nature 400: 61-63.

Stoll, P. and Weiner, J. 2000. A neighborhood view of interactions among individual plants. - In: Dieckmann, U. et al. (eds), The geometry of ecological interactions: simplifying spatial complexity. Cambridge Univ. Press, pp. 11-27.

Tilman, D. 1982. Resource competition and community structure. - Princeton Univ. Press, pp. 296.

Tilman D. 1994. Competition and biodiversity in spatially structured habitats. - Ecology 75: 2-16.

Turnbull, L. A. et al. 2000. Are plant populations seed-limited? A review of seed sowing experiments. - Oikos 88: 225-238.

Uriarte, M. et al. 2010. Trait similarity, shared ancestry and the structure of neighbourhood interactions in a subtropical wet forest: implications for community assembly. - Ecol. Lett. 13: 1503-1514.

Warton, D. I. and Hui, F. K. C. 2010. The arcsine is asinine: the analysis of proportions in ecology. - Ecology 92: 3-10.

Wright, M. G. and Samways, M. J. 2011. Plant characteristics determine insect borer assemblages on Protea species in the Cape Fynbos, and importance for conservation management. - Biodivers. Conserv. 8: 1089-1100.

Yates, C. J. et al. 2010. Projecting climate change impacts on species distributions in megadiverse South African Cape and Southwest Australian Floristic Regions: opportunities and challenges. - Aust. Ecol. 35: 374-391. 